\title{
A Novel UWB Monopole Antenna with Reconfigurable Band Notch Characteristics Based on PIN Diodes
}

\author{
Yahieal Alnaiemy, and Lajos Nagy
}

\begin{abstract}
Our design for a novel UWB monopole antenna structure with reconfigurable band notch characteristics based on PIN diodes is presented in this paper. The proposed antenna is comprised of a modified circular patch and a partial ground plane. The band-notch characteristics are achieved by etching a slot on the partial ground plane and inserting three PIN diodes into the slots for adjusting the operating antenna bands. The reconfigurability is achieved by adding three PIN diodes to obtain eight states with UWB, dual and triple operating bands which can be obtained by changing the PIN state from ON to OFF, and vice versa. The proposed design shows a simple biasing process to switch the frequency bands with insignificant gain variation and low radiation efficiency reduction. The reconfigurability of the frequency is accomplished by adjusting the effective slot length through modifying the PIN diodes states at the desired operating bands. The desired operating frequency bands can be obtained by switching the diodes. A systematic parametric study based on a numerical analysis is invoked to verify and refine the proposed performance. The proposed antenna is fabricated on FR-4 substrate with dimensions of $50 \times 60 \times 1 \mathrm{~mm}^{3}$. The proposed antenna performance was tested experimentally and compared to the simulated results from CSTMW based on FIT. Experimental results were in concordance with simulated results. We found that the proposed antenna design had simple geometry and it was easy to control the frequency bands to suit the applications of WiMAX and WiFi systems.
\end{abstract}

Index Terms-UWB, Notch band, Reconfigurable antenna, PIN diode, Monopole antenna.

\section{INTRODUCTION}

D UE to the rapid growth in wireless communication technology, multi-band antennas are highly valued. A significant drawback of conventional multi-band antennas is that they work only for specific operations. A new antenna with desired frequency specifications is required for each new application, increasing the relative cost. Reconfigurable antennas are usually based on active electronic devices to independently tune the required frequency, radiation, and polarization. They have come into urgent demand due to the growth in mobile communication technologies[1]. Reconfigurable antennas have several advantages over conventional antennas such as smaller size, steerable radiation patterns, selecting the desired polarization for different frequency bands,

Yahiea Alnaiemy is with Budapest University of Technology and Economics, Budapest, Hungary, and University of Diyala, College of Science e-mail: (yahiea@hvt.bme.hu).

Lajos Nagy is with the Budapest University of Technology and Economics, Department of Broadband Infocommunications and Electromagnetic Theory, Budapest, Hungary e-mail: (nagy.lajos@vik.bme.hu).

Manuscript received July1, 2021; revised July 30, 2021.

DOI: 10.36244/ICJ.2021.3.4 and polarization, which reduces antenna system size and intersymbol interference (ISI) impacts [2]. The integration of these configurations into a single antenna is a major challenge that researchers have been faced in recent years. Integrating antennas with modern high-speed semiconductors such as Positive Intrinisic Negative (PIN) diodes, Radio Frequency Microelectromechanical Systems (RF MEMS), and varactor diodes have been designed successfully for frequency, polarization, and pattern tunability [3]-[25]. Due to the highspeed response and low forward resistance of PIN diodes, it was common practice to vary the antenna performance [3] for direct antenna modulation (DAM) including Differential Phase Shift Keying (DPSK) modulation [4], which makes it more competitive for cognitive radio applications. A slotted patch antenna with two PIN diodes was presented by Majid et $a l$ for frequency and pattern reconfiguration [5]. For antenna beam reconfiguration, a flexible antenna based on eight PIN diodes for wireless applications was introduced by Zhu et al in [6]. For frequency and pattern reconfiguration, an antenna based on two PIN diodes positioned within the slot etched in the front antenna patch was presented by Han et al in [7] for Long-Term Evolution (LTE). A reconfiguration of the antenna frequency and pattern was achieved using a printed antenna based on three Radio Frequency (RF) switches [8]. A reconfigurable Ultra-Wide-Band (UWB) filter antenna was featured in [9]; controlling operating frequency bands based on three PIN diodes that directly control the desired WLAN and WiMAX frequency bands. Wu et al in [10] introduced and designed a reconfigurable quad-band antenna based on Micro-Electomechanical-Systems (MEMS) switches. Controlling operating frequency can be obtained by adjusting the MEMS switch, making it suitable for the cognitive radio base station. Hamid et al in [11] discussed and modelled a Vivaldi antenna by inserting four switchable ring slots within the antenna ground plane structure. The antenna reconfiguration may work in broadband or narrowband mode by changing the PIN diode switching facility. For single, multi-band, and UWB spectrum, a frequency reconfigurable microstrip antenna was presented by Yadav et al in [12], based on an array of 27 PIN diodes integrated into the partial ground structure. Yadav et al used many RF switches, increasing cost, manufacturing, and measurement difficulties relative to our antenna design. Tasouji et al in [13] presented a printed UWB slot antenna based on two PIN diodes with reconfigurable band-notch features that was mounted across the circular slot antenna patch to produce single and double band-notch characteristics. A UWB 
A Novel UWB Monopole Antenna with Reconfigurable

Band Notch Characteristics Based on PIN Diodes monopole antenna with a reconfigurable band-notch based on two PIN diodes placed in antenna patch slots was presented by Han et al in [14]. A reconfigurable microstrip slot antenna was presented by Oraizi et al in [15] through regulation the PIN diode embedded in the rectangular Split Ring Resonator (SRR). A reconfigurable UWB circular wide-slot antenna based on a stepped impedance resonator and an arc-shaped parasitic element was presented by Li et al in [16]. A notchedband UWB monopole antenna was introduced by Aghdam et al in [17] by connecting a varactor diode to a $\pi$-shaped patch. A reconfigurable cavity-backed slot antenna substrate integrated waveguide based on MEMS active elements was presented by Saghati et al in [18]. A dual-band reconfigurable antenna based on a varactor diode lumped into the slot antenna was introduced by Behdad et al in [19]. A reconfigurable circular monopole based on Field Effect Transistor (FET) was presented in by Aboufoul et al in [20] for cognitive radio applications. A reconfigurable frequency band monopole with single and dual bands by employing three PIN diodes was presented by Shah et al in [21]. Nikolaou et al in [22] controlled the frequency resonance by switching two PIN diodes soldered on both sides of an annular slot patch antenna. Kim et al in [23] were proposed the polarization reconfigurability of a single feed circular patch antenna with five PIN diodes for low frequency and high frequency applications. Elwi in [24] presented a reconfigurable antenna with remotely controlled by integrating a photo resistor array into a Hilbert patch antenna and adjusting the photo-resistors elimination for modern 5G applications. Singh et al in [25] were proposed a reconfigurable antenna for tuning the frequency bands in internet of things (IoT) systems using three PIN diodes. The researchers for wireless applications have presented several wideband monopole antennas. For instance, Reddy et al in [26] designed a flexible wideband monopole antenna for body-centric wireless communications. While, Mohandoss et al in [27] designed a planar monopole fractal antenna to enhance the bandwidth for the personal wireless area and UWB applications. A multiband reconfigurable microwave filtering monopole antenna was presented by Kingsly et al in [28] based on switchable agile multiband filtenna for cognitive radios and Time Division Multiple Access (TDMA) systems. Finally, compact wideband flexible planar monopole antennas were designed and analyzed for body-centric wireless and UWB communications [29].

The main objective of using such reconfigurable UWB frequency antenna is to overcome the overlap of the UWB with other bands, such as WiMAX bands. The antenna performances are obtained from a comparative study of the conventional antenna with the proposed antenna based on PIN diodes within the ground plane. This paper introduces a well-controlled operating frequency band based on three $O N-O F F$ switch statues. By changing the slot length effectively through the PIN diode switching, a frequency band reconfiguration can be achieved at the desired operating bands accordingly. The proposed antenna shows a frequency band from $1.86 \mathrm{GHz}$ to $10.89 \mathrm{GHz}$ that is significantly affected by PIN diodes switching. Eight cases, therefor, can be generated from switching the proposed three PIN diodes to provide
UWB, two, and/or three operating bands. The simulated results are compared to the experimental results, which show acceptable agreement and confirm good performance of the proposed antenna. The obtained results, therefore, confirm that the proposed reconfigurable antenna is a better candidate for integration into wireless communication circuits.

\section{Antenna Design Detials}

The proposed antenna is designed to operate from 1.89 to $10.89 \mathrm{GHz}$ to achieve a UWB with matching impedance in terms of $S_{11}$ bellow $-10 \mathrm{~dB}$ according to the IEEE standard [15]. To be suitable for near and medium communication distance, the antenna's peak realized gain should be within the range of $5.2 d B-6.03 d B$ for $\mathrm{WiFi}$ applications. The proposed antenna design, with the suggested parameters, is depicted in the next section. The antenna is modeled step by step to obtain a UWB response with an impedance bandwidth of $\left(S_{11}<-10 \mathrm{~dB}\right)$ for the entire UWB (1.85-10.9) $\mathrm{GHz}$. Next, three PIN diodes are connected to the ground plane slot as switches to obtain eight PIN diode states. The main reason to choose three PIN diodes as switches is to change the proposed antenna's effective electrical length to achieve frequency reconfigurability. By switching the state of the PIN diode between forward and backward, we can achieve the proposed antenna design to operate in UWB, dual, and tripleband mode.

\section{Methodology of the Antenna Design}

In this section, we present the geometrical details of the proposed antenna to provide the design methodology for the optimal antenna performance. Next, antenna reconfiguration is discussed by switching the PIN diodes. The reconfigurability of the antenna can be accomplished by employing three PIN diodes as switches in the simulation setting to obtain dual-band mode, triple-band mode and UWB depending on the switch state. The authors have chosen a proposed monopole antenna, based on the circular shape and a defected ground structure that could expand the frequency band of operating as can be seen later in this paper.

\section{A. UWB Monopole Antenna Design}

The configuration of the proposed UWB monopole antenna geometry with the design parameters is illustrated in Fig. 1 and Table I, respectively. The proposed antenna is fabricated on a low-cost FR4 substrate with a $\epsilon_{r}$ of 4.3 , and a $\tan \delta$ of 0.025 . The proposed antenna size is $50 \mathrm{~mm} \times 60 \mathrm{~mm}$ with a substrate thickness of $1 \mathrm{~mm}$. The modified circular patch is printed on the top side, and the defected partial ground plane is printed on the bottom side. The radiating patch and ground plane shapes are modified to achieve a UWB with good impedance matching.

In this section, reach the final antenna design is discussed. The antenna design methodology was proposed by using a commercial software package of Computer Simulation Technology Microwave Studio (CSTMW) with Finite Integration Technique (FIT) algorithm [30]. For this, the antenna bandwidth is monitored to get the best matching impedance over 


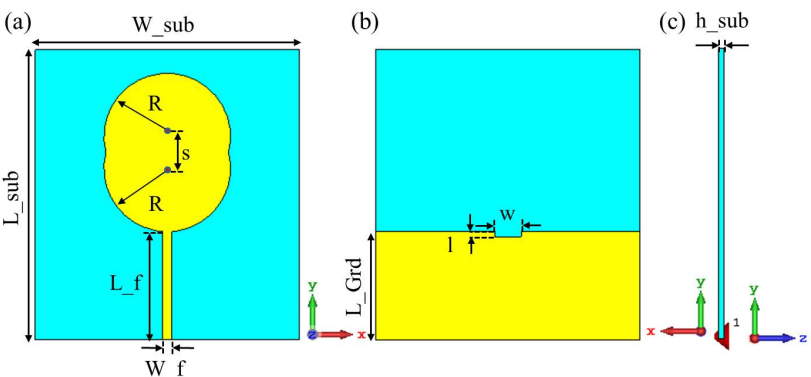

Fig. 1. The proposed antenna geometry; (a) front view, (b) back view and (c) side view.

TABLE I

THE GEOMETRICAL DIMENSIONS OF THE PROPOSED ANTENNA: ALL DIMENSIONS IN $\mathrm{mm}$.

\begin{tabular}{|c|c|c|c|}
\hline Parameter & Dimension & Parameter & Dimension \\
\hline$L \_s u b$ & 60 & $L \_f$ & 23 \\
\hline$W \_s u b$ & 50 & $W \_f$ & 1.65 \\
\hline$L \_G r d$ & 23 & $R$ & 12 \\
\hline$h \_s u b$ & 1 & $S$ & 6 \\
\hline$w$ & 5 & $l$ & 1 \\
\hline
\end{tabular}

the entire band of interest by feeding the antenna with a $50 \Omega$ port. To determine the influence of changing antenna dimensions, a parametric study was conducted. Antenna performance was monitored with respect to changing $W_{f}, W, l$, $S, h \_s u b$, and $L \_G r d$ parameters representing feed line width, slot width of the partial ground plane, slot height of the partial ground plane, center-to-center distance, substrate height, and ground plane length, respectively. Next, a parametric study was applied to find the appropriate substrate type and size. The antenna design begins with a conventional circular patch backed by a full ground plane as mentioned in case 1 (Fig.2). Next, the ground plane is changed to a partial ground plane (without any slots) to improve the impedance matching, as mentioned in case 2. Next, the rectangular slot is etched from the ground plane to improve the proposed antenna bandwidth. The frequency reconfiguration is accomplished by modifying the parasitic element electrically through switching the PIN diodes with the ground plane. The defected ground plane is made by integrating the PIN diodes without changing the UWB antenna performance. We observed that the desired UWB results are not obtained for the proposed antenna with partial ground plane, therefore, a slot is inserted within the partial ground plane to improve the bandwidth and the matching impedance. We observed that the antenna bandwidth of $3.5 \mathrm{GHz}$ to $9.5 \mathrm{GHz}$ was obtained as mentioned in case 3 . A further modification of the proposed antenna is to involve a circular patch as denoted in case 4 of Fig. 2. The modification in this design includes another circular shape to generate a new frequency band. This modification in the radiator patch obtains a better impedance matching over the entire UWB and enhances the reference antenna bandwidth as shown in case 4 . We observed a very wide bandwidth $\left(S_{11}<-10 \mathrm{~dB}\right)$ of 1.85 $\mathrm{GHz}$ to $11 \mathrm{GHz}$.

It is indicated from the $S_{11}$ spectra Fig. 2 that variation in

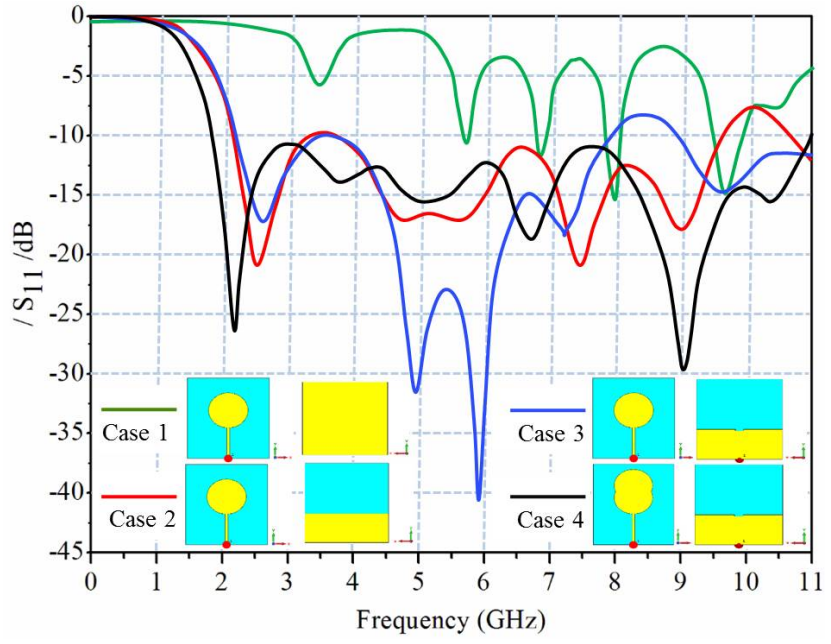

Fig. 2. Comparison of simulated $\left|S_{11}\right|$ spectra for four model cases.

the ground plane has significant effects on the antenna bandwidth. For example, the proposed antenna with full ground plane in case 1 shows narrow bandwidth around multiple frequency bands. Antenna bandwidth, therefore, is enhanced when a partial ground plane is modified. However, the antenna bandwidth is enhanced furthermore in cases 3 and 4 when the slot is introduced to the ground plane. The results are in agreement with the suggested antenna design's requirements which are provided in Section II for achieving a wide-band with matching impedance below $-10 d B$.

To maximize the antenna bandwidth further, the antenna feed line was connected to the Sub Miniature version A connector (SMA) center pin with a width of $W_{f}$. The feed line width is parametrically optimized as shown in Fig.3. It is indicated that varying $W_{f}$ shows a major impact on the antenna bandwidth [24]. This study may lead to the best impedance matching and subsequently, a gradual bandwidth increase in the proposed antenna. Figure 3 shows the optimized results of $W_{f}$ at 1.65 $\mathrm{mm}$ with broader bandwidth over the frequencies from 1.85 $\mathrm{GHz}$ to $10.89 \mathrm{GHz}$. This parametric study considers $S_{11}<$ $-10 \mathrm{~dB}$ impedance bandwidth for the entire UWB by changing $W_{f}$ given the antenna wide band from 1.85 to $10.85 \mathrm{GHz}$.

The main purpose of the rectangular slot on the ground plane is to disturb the surface current to operate the antenna at lower frequency bands. The effects of changing the slot width $(W)$ on the $\left|S_{11}\right|$ spectra (Fig. 4) was studied to realize the best matching impedance. We found that decreasing the slot width $(W<5 \mathrm{~mm})$ causes impedance matching reduction from $2.5 \mathrm{GHz}$ to $7.8 \mathrm{GHz}$ in the low-frequency band, while an increase in slot width $(W>5 \mathrm{~mm})$ creates problems with impedance matching from 7.2 $\mathrm{GHz}$ to $7.8 \mathrm{GHz}$ in the high-frequency band. The maximum impedance bandwidth and best impedance matching can be obtained when $W=5$ $m m$. A sweep of the parameter $W$ to achieve the proposed antenna work with a UWB mode with impedance bandwidth less than $-10 d B$ from $1.85 \mathrm{GHz}$ to $10.9 \mathrm{GHz}$.

To maximize the antenna bandwidth, the height of the slot 


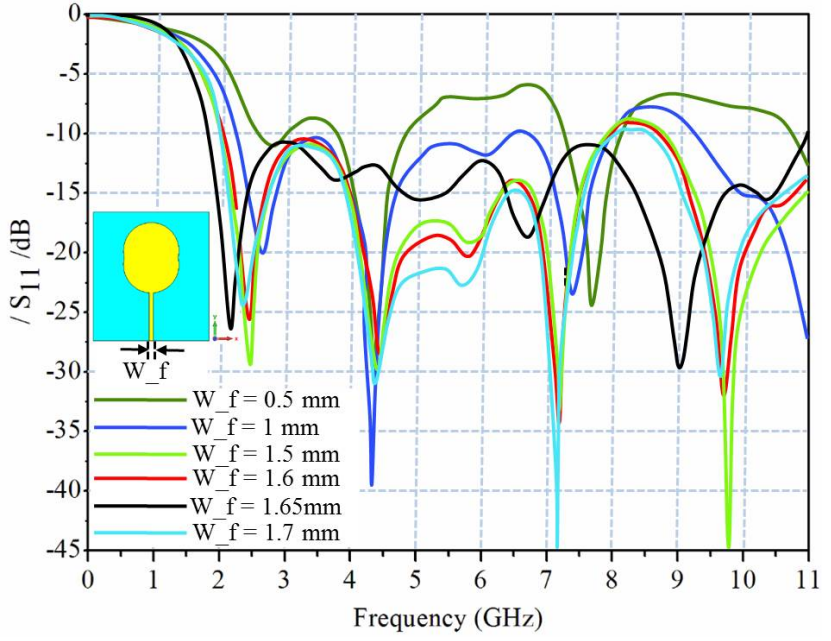

Fig. 3. Effects of changing $\left(W_{f}\right)$ on the $\left|S_{11}\right|$ spectra

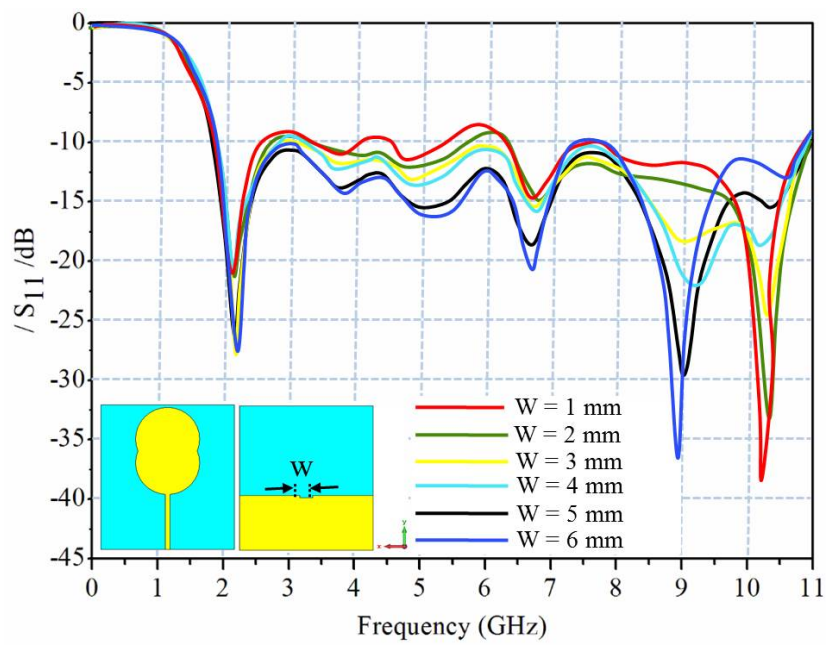

Fig. 4. Effects of changing $W$ on the $\left|S_{11}\right|$ spectra

on the partial ground plane has a pivotal function. However, We studied the effect of the slot height of the partial ground plane given by $(l)$ on the proposed antenna performances. The effect on bandwidth for different ground plane slot heights is shown in Fig. 5.

As shown in Fig. 5, the narrower slot height $(l=0.5 \mathrm{~mm})$ indicates poor return loss at the $2.9 \mathrm{GHz}$ to $3.2 \mathrm{GHz}$ band. Increasing the slot height $(l>1 \mathrm{~mm})$ leads to enhanced $\left|S_{11}\right|$ at the lower band (1.75-6.75) $\mathrm{GHz}$, but poor impedance matching is found between $6.75 \mathrm{GHz}$ and $8.5 \mathrm{GHz}$. However, the optimum ground slot height $(l=1 \mathrm{~mm})$ provides the necessary impedance matching over the required frequency range, and the UWB width of the antenna is from 1.75 to $11 \mathrm{GHz}$. These results were extremely close to the planned antenna specification, as shown in Section II. The length of partial ground plane (L_Grd) shows a slight effect on the proposed antenna bandwidth. The $L \_G r d$ was swept from 20 $\mathrm{mm}$ up to $25 \mathrm{~mm}$ with a step of $1 \mathrm{~mm}$ to obtain the desired UWB frequency band. We observed from Fig. 6 that the

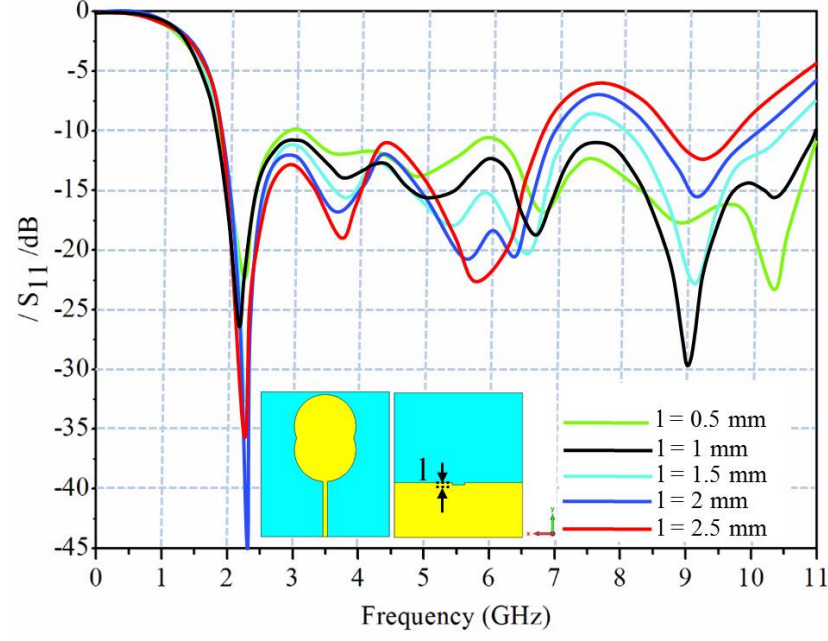

Fig. 5. Effects of changing $l$ on the $\left|S_{11}\right|$ spectra

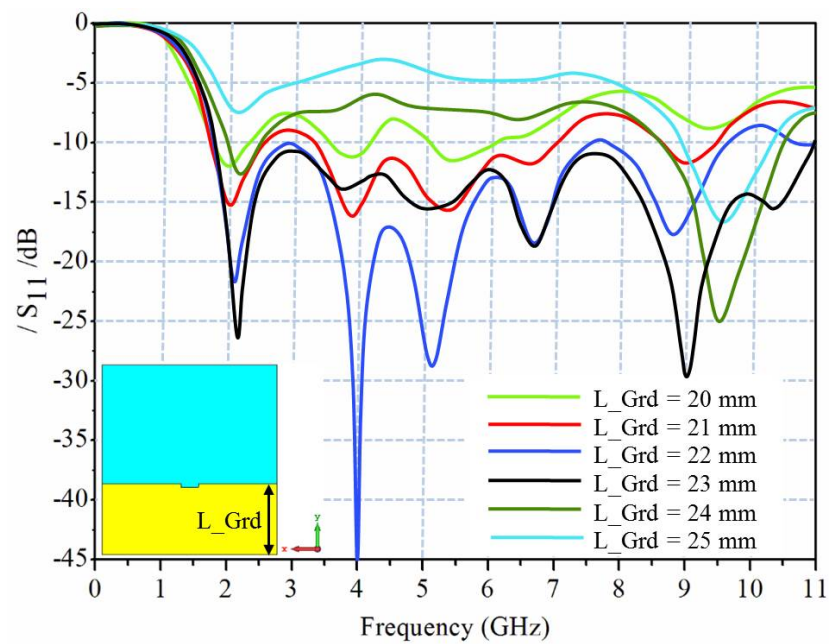

Fig. 6. Effects of changing $L_{-} G r d$ on the $\left|S_{11}\right|$ spectra

proposed antenna operates as a UWB antenna when $L \_G r d=$ $23 \mathrm{~mm}$. However, $\left|S_{11}\right|$ is improved dramatically when the ground plane length is gradually reduced. As a result, the required antenna design criteria are met by selecting $L \_G r d$ $=23 \mathrm{~mm}$ for the entire UWB mode with the best bandwidth impedance matching less than $-10 d B$.

Figure 7 shows the circular patch development through five stages to illustrate the effects of changing $S$ value from $0 \mathrm{~mm}$ to $8 \mathrm{~mm}$ with a step of $2 \mathrm{~mm}$. The parameter $S$ represents a center-to-center distance between two circular patches ( Fig.1). In this design step, the simulated $\left|S_{11}\right|$ spectra of the proposed antenna with a different value of $S$ is illustrated in Fig. 7.

At a low value of $S(S<6 \mathrm{~mm})$, the antenna does not provide a UWB impedance bandwidth. The proposed antenna shows poor matching impedance in the high- frequency band from $7.2 \mathrm{GHz}$ to $8.1 \mathrm{GHz}$. Increasing $S$ above $6 \mathrm{~mm}$, improves the impedance bandwidth. However, the antenna still suffers from a matching impedance problem at the frequencies from $7.3 \mathrm{GHz}$ to $8.3 \mathrm{GHz}$. The distribution of the surface 


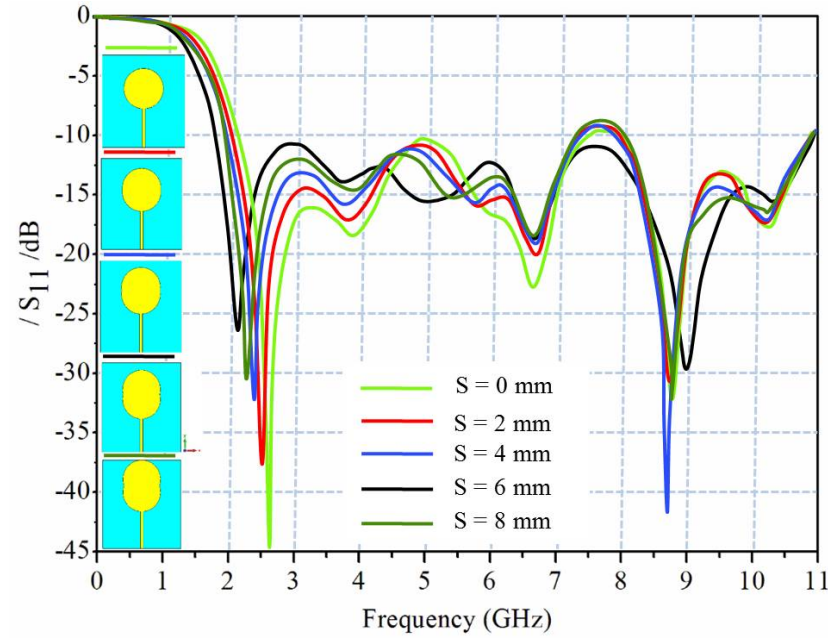

Fig. 7. Simulated $\left|S_{11}\right|$ spectra for the proposed patches.

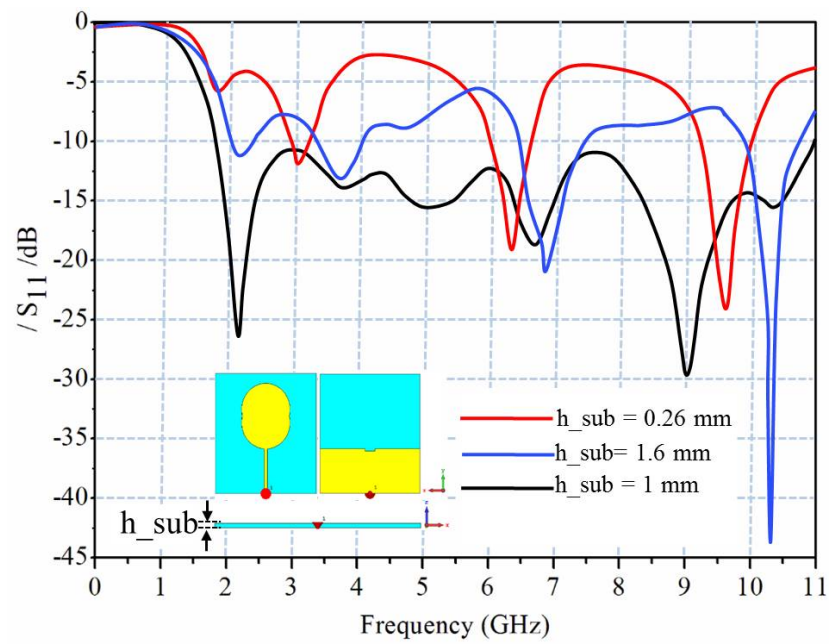

Fig. 8. Effects of changing $h_{-} s u b$ on the $\left|S_{11}\right|$ spectra.

current of the proposed antenna with $S=6 \mathrm{~mm}$ is mainly extended. By considering $S=6 \mathrm{~mm}$, therefore, the impedance matching bandwidth is significantly enhanced to suit the proposed antenna requirement to work with UWB mode.

Figure 8 demonstrates the effects of changing the substrate thickness on the $\left|S_{11}\right|$ spectra. The substrate thickness was changed according to the available commercial resources $: 0.26,1$, and $1.6 \mathrm{~mm}$. Based on the $\left|S_{11}\right|$ spectra, the proposed antenna shows a wider impedance bandwidth for $h \_s u b=$ $1.6 \mathrm{~mm}$ but cannot support the desired UWB. The proposed antenna depicts a wide impedance bandwidth only when it has substrate thickness of $1 \mathrm{~mm}$. As a result, if $h_{-} s u b=1 \mathrm{~mm}$, the proposed antenna operates in UWB mode with a matching impedance of less than $-10 d B$ from 1.85 to $10.9 \mathrm{GHz}$.

Additionally, we discuss the effects of substrate type change on antenna performance. As shown in Fig. 9, FR-4 substrate with $\epsilon_{r}=4.3$ and $\tan \delta=0.025$ covers a wider bandwidth compared to other substrates in this study, which satisfied the entire UWB.

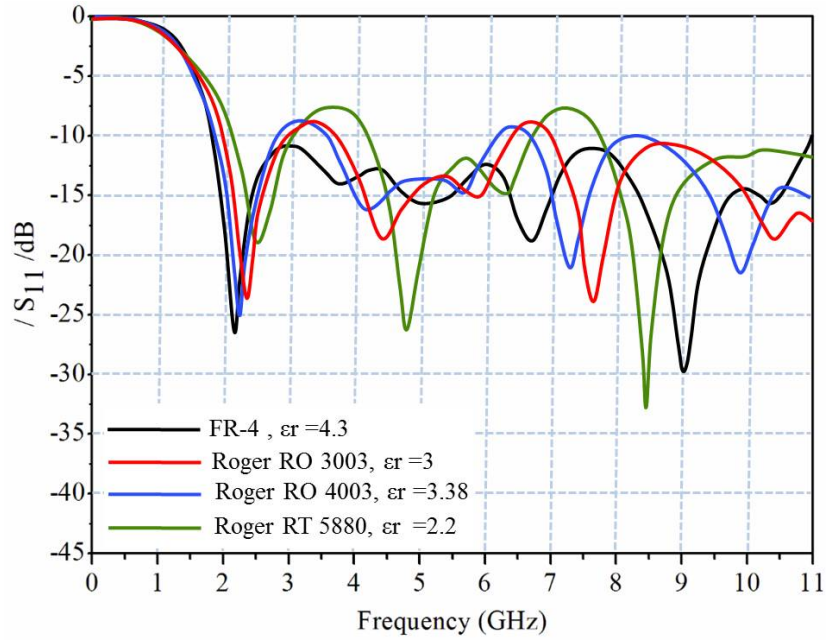

Fig. 9. Effects of changing substrate materials on the $\left|S_{11}\right|$ spectra.

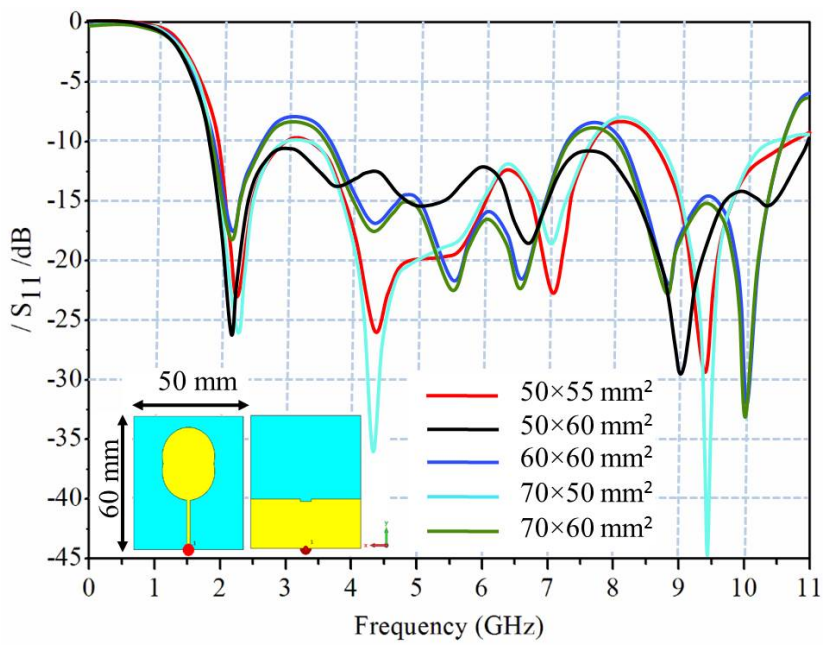

Fig. 10. Effects of substrate size on the $\left|S_{11}\right|$ spectra.

Regarding substrate size, we adjusted the proposed antenna dimensions within five different dimensions. As shown in Fig. 10 , we found the best antenna dimensions are $50 \mathrm{~mm} \times 60$ $m m$, which satisfied the entire UWB.

\section{B. Reconfigurable UWB Monopole Antenna}

The reconfiguration of the proposed antenna, which has a slot on the ground plane, is shown in Fig.11(a). The slot on the ground plane is mounted by a narrow a metal strip with dimension $0.5 \mathrm{~mm} \times 4 \mathrm{~mm}$ to provide independent DC biasing for PIN diodes as shown Fig.11(a). The frequency configuration is changed by adjusting three PIN diodes connected to the ground plane slot. In the RF domain, PIN diodes are commonly used, therefore, the PIN diode RF resistance is connected to the DC bias current and can be used as an RF switch. When the diode is positively biased, the short circuit is turned on, and the open circuit is turned $\mathrm{ON}$ when it is reverse biased. Usually, the diode resistance 
A Novel UWB Monopole Antenna with Reconfigurable

Band Notch Characteristics Based on PIN Diodes can vary from $10 k \Omega$ to less than $1 \Omega$ by controlling its bias current [32]. In UWB mode, switching the PIN diodes may dramatically affect the impedance matching that reduces the bandwidth due to parasitic inductance, capacitance, and resistance. Nevertheless, the DC biasing circuit is necessary to control the RF signal flow to the antenna structure. For this, integrating the PIN diodes into the ground plane has minimum effect compared with maximum loading effects on the antenna performance when the PIN diodes are integrated within the radiating patch [31]. We prefer to mount the PIN diodes and associate the biasing circuit in the ground plane instead of the patch antenna due to the biasing complexity and the antenna radiation interfering with the antenna structure [31]. The main reason for choosing three PIN diodes as switches is to change the proposed antenna effective electrical length to achieve the proposed frequency reconfigurability. The defected partial ground plane resonators self-resonance frequencies depend on their physical dimensions. The defected partial ground plane operation is very similar to a circuit with parallel inductance capacitance [8]. Through an increase in the overall length of the ground plane slot, the inductance could be raised, while reducing the slot width increases the capacitance. The notched band frequency can be approximated using equation 1 [32].

$$
f_{\text {notch }}=\frac{c}{4\left(L \sqrt{\epsilon}_{e}\right)}
$$

Here, the overall length of the defected partial ground plane slot is $L, \epsilon_{e}$ is the effective dielectric constant, and $c$ is the light speed in the free space.

In the proposed configuration, the slot width $(W)$ and slot length $(l)$ are fixed to $5 \mathrm{~mm}$ and $1 \mathrm{~mm}$, respectively. The resonant frequency can be controlled using the overall defected partial ground plane slot length through the PIN diodes. The proposed antenna depicts a dual and triple notch band only when the PIN diodes are inserted within the ground plane slot. The three PIN diodes are used to adjust inductance and capacitance of the antenna equivalent circuit. This variation contributes to the tuning of impedance matching in desired operating bands and optimizing the corresponding resonant circuitry. Figure 11(b, c, e, and d) indicates the equivalent circuits for the ON and OFF conditions.

To explain the PIN diode operation (ON-OFF), an ideal PIN diode is used as a switch in the simulation, and the proposed diodes are modelled as a metal strip only for direct open and short states. Under ON status, the respective circuit shows that the inductor $(L)$ and a resistor $\left(R_{S}\right)$ are loaded in series connections, while an inductor $(L)$ is loaded in series with a capacitor $\left(C_{T}\right)$ when the PIN is switched to OFF. In this analysis we used a suitable bias to prevent the coupling of the RF signal and the bias current [31]. Capacitors are utilized to block DC and pass RF signal. Inductors are used as chokes to block RF signal and pass DC as seen in Fig.11(d). We observed that it is possible to switch the proposed reconfigurable antenna between a UWB mode, dual band-notch modes and a triple band-notch mode. Figure 12 presents the $\left|S_{11}\right|$ spectra and the realized gain versus frequency of the proposed antenna based on the eight diode states. State 1 is establishing the UWB (1.86-10.89) $\mathrm{GHz}$ when all the PIN diodes are OFF.
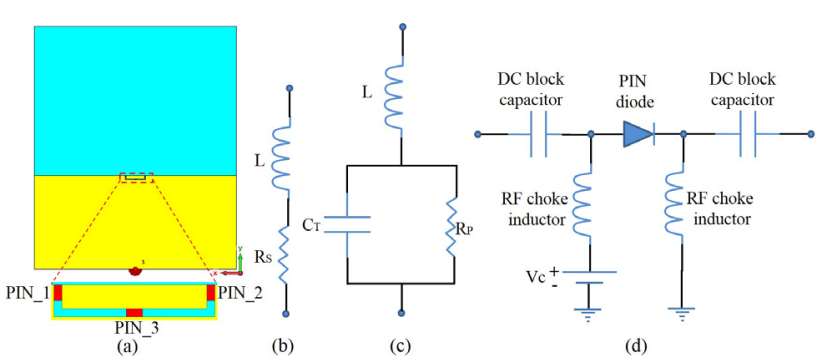

(a)

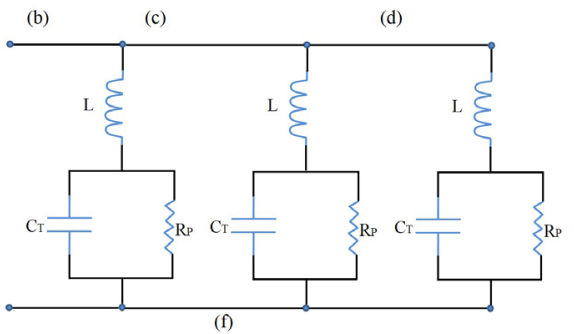

Fig. 11. The proposed PIN diodes and bias circuit (a) proposed reconfugrable antenna, (b) equivalent circuit for ON state of one PIN diode, (c) equivalent circuit for OFF state of one PIN diode, (d) bias circuit for PIN diodes, (e) equivalent circuit for ON state of all PIN diodes, and (e) equivalent circuit for OFF state of all PIN diodes,

States 4, 6, and 8 establish the dual bands, and states 2, 3, 5, and 7 are solely responsible for obtaining the triple bands. The transition between dual, triple and UWB can be explained by the change in the surface current. Indeed, the electrical length of the proposed antenna structure is really determined by the diodes condition (ON and OFF). From the proposed antenna $\left|S_{11}\right|$, the antenna bandwidths are significantly affected by the PIN diode switching. It can be inferred that the parasitic element affects the antenna bandwidth to be changed from the UWB to the narrow bands. Due to parasitic effects, the proposed UWB mode is decreased to narrowband mode. The bandwidth is changed from UWB to narrow band due to the difference in surface current distribution [16]. In fact, the proposed antenna electrical length can be determined from switching PIN diodes conditions as summarized in Table II. We observed from the realized antenna gain versus frequency plot in which frequencies the UWB notch antenna radiates and where it does not. The obtained gain is found to be suitable for short and medium wireless applications [7]; ranging from $5.2 d B$ to $6.1 d B$ as shown in Fig. 12(b).

TABLE II

SUMMARY OF THE PIN DIODES SWITCHING STATES MODES

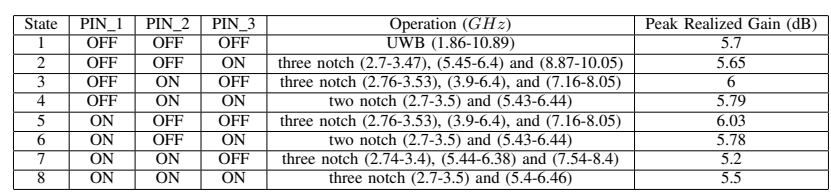

\section{Fabrication, Measurements, and Testing}

The proposed antenna is fabricated calculating the optimal design. The fabrication is performed in the printed wiring board laboratory of the BME-ETT, Department of Electronic 


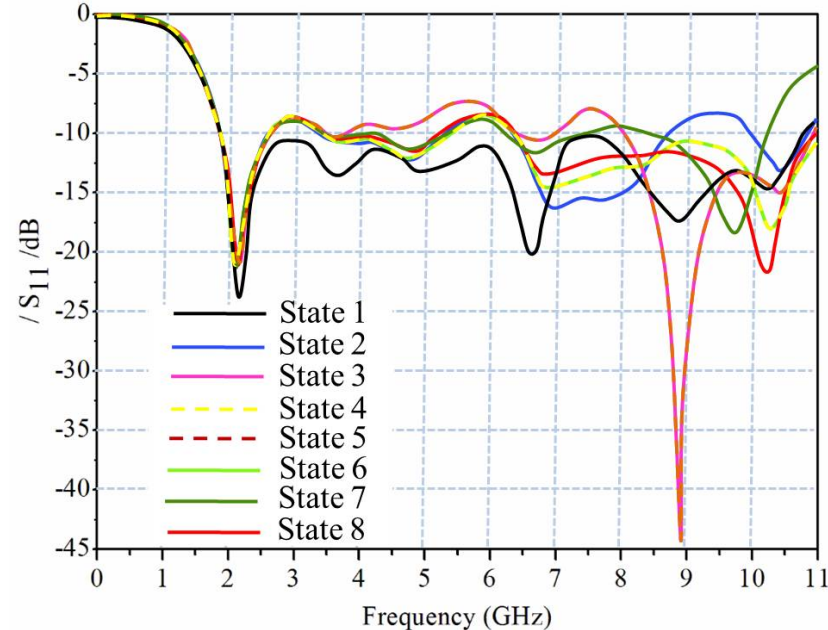

(a)

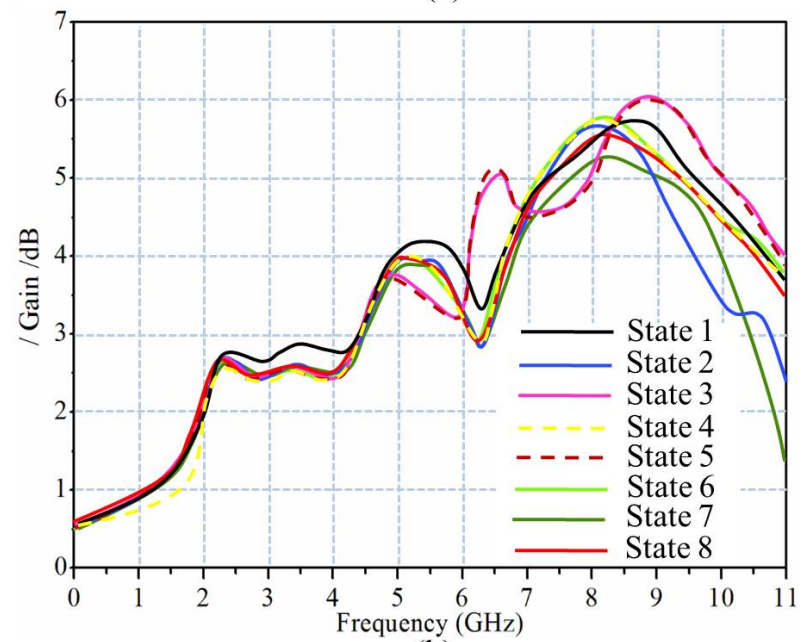

(b)

Fig. 12. Simulated (a) reflection coefficient Vs frequency, and (b) realized gain Vs frequency for the all the PIN diodes states.

Technology, Budapest University of Technology and Economics. The Printed Circuit Board (PCB) technique is used to fabricate the proposed antenna on low-cost FR-4 substrate with a dielectric constant of 4.3 , a loss tangent of 0.025 , and substrate thickness of $1 \mathrm{~mm}$. The fabricated antenna is fed through an SMA connector. BAR6303W PIN diodes are utilized as high-speed RF-signal switches [33]. Different shapes of the slotted ground plane connect to the PIN diodes. Four reconfigurable antennas are fabricated based on the information presented in Fig. 1. To identify the characteristics of the band notch, the PIN diodes are soldered within the slotted ground plane as shown in Fig. 13. The experimental measurements are tested using Vector Network Analyzer (VNA) STAR ms4642A Series inside an RF anechoic chamber. By using VNA ranging (1 M Hz-14 GHz), the return losses of the fabricated antenna are measured and tested to validate the simulated results with the measured ones. Due to the symmetry of the PIN diode states $(2,3,5$, and 7$)$, we fabricated only state 3 by integrating PIN_2 within the slotted ground plane and measured the

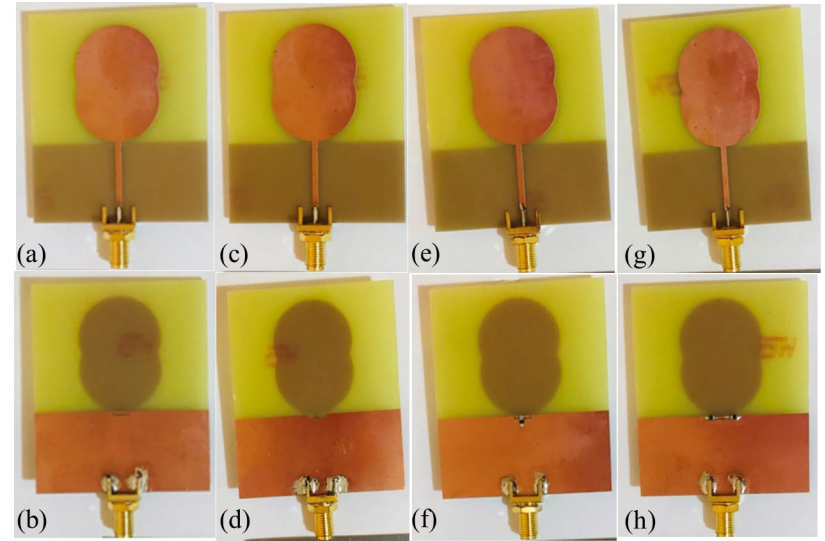

Fig. 13. The proposed antenna fabricated (a), (c) front view without PIN diodes and (e), (g) front view with PIN diodes, and (b), (d) back view without PIN diodes and (f), (h) back view with PIN diodes.

corresponding $\left|S_{11}\right|$. Because of the symmetry among states 4,6 , and 8 , we fabricated only state 6 by integrating PIN_1 and PIN_3 within the slotted ground plane and measured the corresponding $\left|S_{11}\right|$ as seen in Fig. 13.

The proposed antenna is connected to a bias tee circuit through the SMA port of $50 \Omega$ input impedance. The PIN diodes are connected to the DC source through the positive negative electrodes that come out from the bias tee to ensure no interference between the DC and the RF sources. In Fig. 14 , the RF source is connected to the antenna and the bias tee electrodes are controlled by a microprocessor Arduino to control the switching process remotely during the measurements.

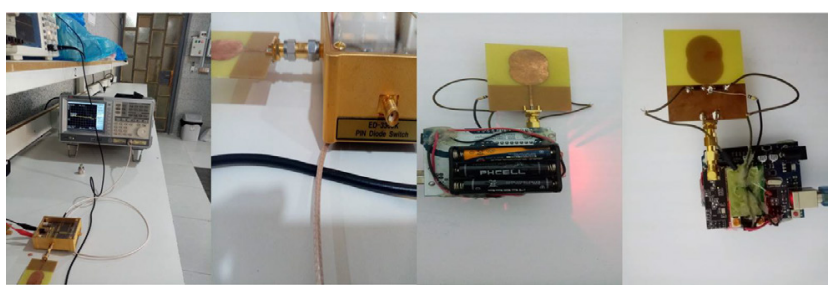

Fig. 14. Proposed antenna measurement setup, biasing circuit for PIN diode.

Antenna performance in terms of $\left|S_{11}\right|$ spectra to all the proposed swiching cases are discussed. Figure 15 shows the simulated and measured $\left|S_{11}\right|$ spectra for the proposed antenna without slots. Figure 16 shows the $\left|S_{11}\right|$ spectra for the proposed antenna with slot on the ground plane but without PIN diodes. We found that the simulated results agree very well with experimental results. The measured results almost agreed with simulated results to support that the proposed antenna provides an UWB response. There is an insignificant difference between simulated and measured results. This deviation is due to certain parameters, such as the manufacturing tolerance, the dielectric permittivity of the substrate, the simulation frequency width, the soldering conditions of the SMA connector, and the measurement circumstances [10].

Next, for the eight switching states, we discuss the first case when all three PIN diodes (PIN_1, PIN_2 and PIN_3) 


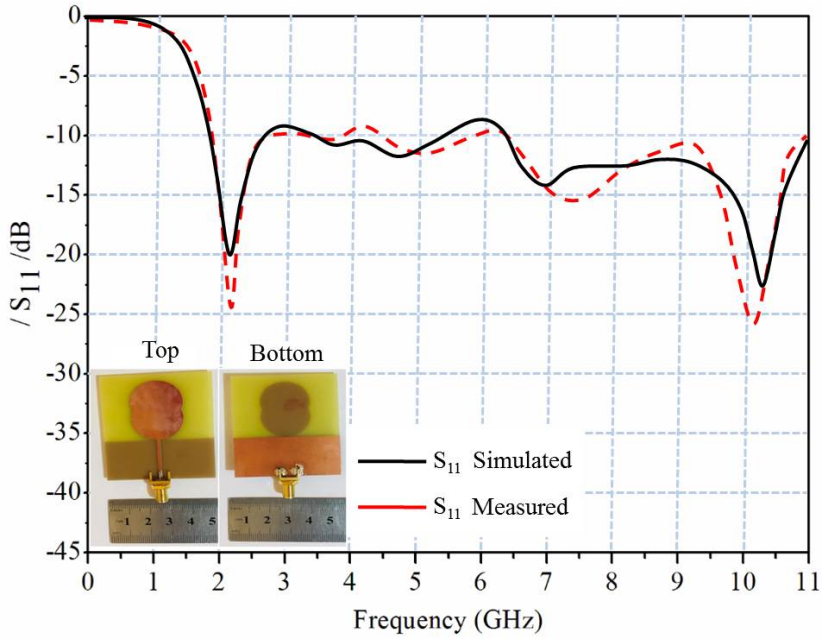

Fig. 15. Simulated and measured $\left|S_{11}\right|$ spectra of the proposed antenna without slot on the ground plane.

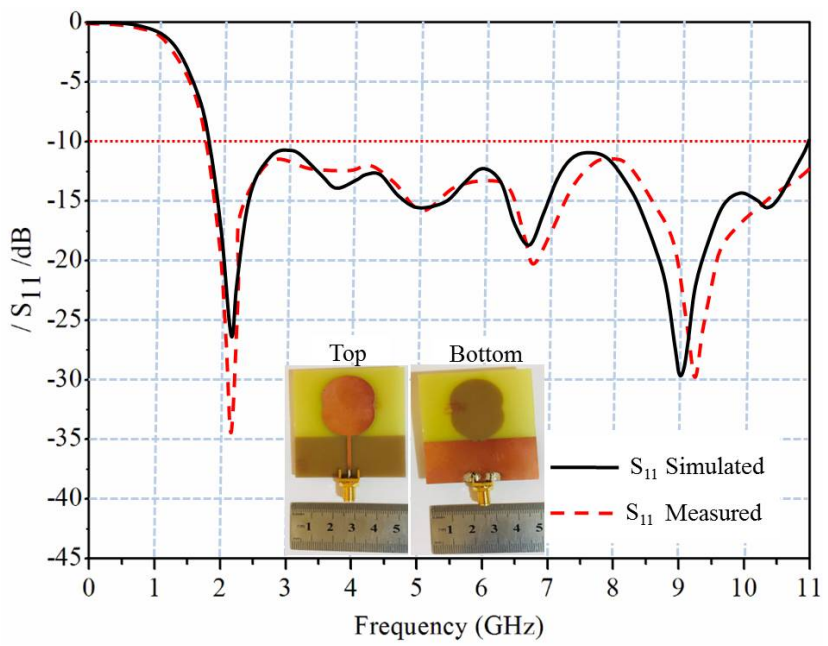

Fig. 16. Simulated and measured $\left|S_{11}\right|$ spectra of the proposed antenna with slot on the ground plane.

are in the OFF state; the proposed reconfigurable antenna operates in UWB mode with a return loss below $-10 \mathrm{~dB}$ from (1.85-10.89 $\mathrm{GHz})$. For simplicity of measurement and to prevent complexity of the connecting wire, the other PIN diodes are not soldered within the proposed antenna. We utilized a separate basis circuit (RF chock and DC block) for each PIN diode, which leads to block DC while passing RF, and RF chokes block RF while passing DC. The antenna is also linked to a Balun structure to avoid interference leakage from the coaxial cable and SMA connector. A good agreement has been obtained between the measured and simulated $\left|S_{11}\right|$ results as seen in Fig. 17.

The next state, when PIN_1 and PIN_2 are in the ON state and PIN_3 is in the OFF state, the proposed reconfigurable antenna operates in triple band mode (2.74-3.4), (5.44-6.38), and (7.54-8.4) $G H z$ with a return loss of $-23 d B,-14 d B$, and $-29.2 d B$, respectively. If PIN_3 is turned ON and the other

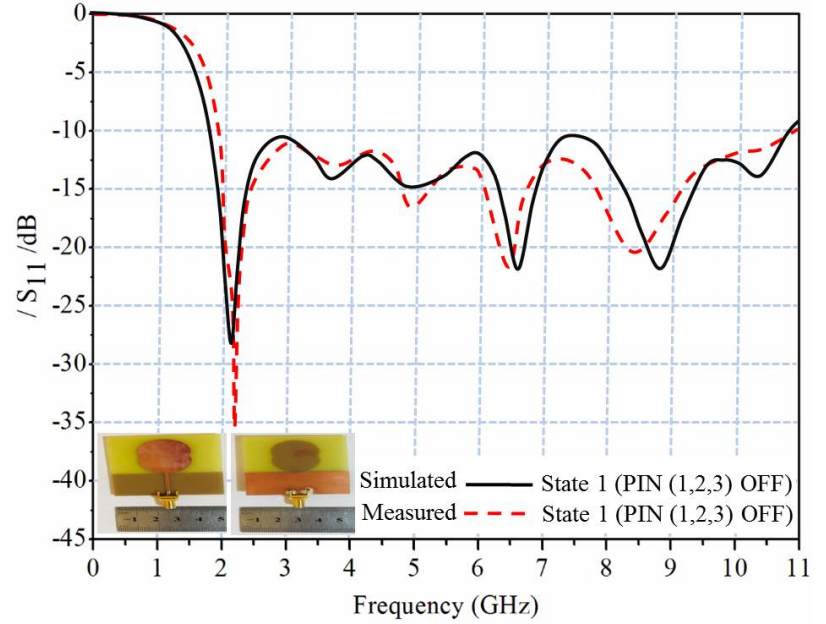

Fig. 17. The simulated and measured reflection coefficient for the operation state 1.

two PIN diodes are turned OFF the proposed reconfigurable antenna operates within three bands (2.7-3.47), (5.45-6.4), and (8.87-10.05) $G H z$ with a return loss of $-23.1 d B,-13 d B$, and $-18.2 d B$, respectively. Figure 18 depicts the measured and simulated $\left|S_{11}\right|$ of the proposed reconfigurable antenna within the three band mode.

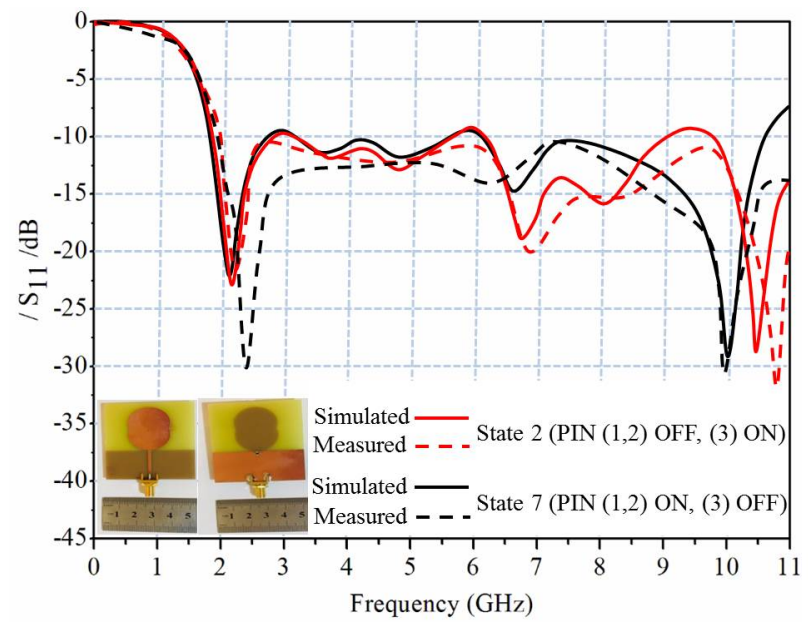

Fig. 18. The simulated and measured reflection coefficient for the operation states 2 and 7.

The next switching states when PIN_1 and PIN_3) are OFF and PIN_2 is ON, and when PIN_2 and PIN_3 are OFF and PIN_1 is ON, the proposed reconfigurable antenna operates in four band mode (1.86-2.75), (3-5.75), (6.15-6.9) and (8.869.75) $G H z$ with a return loss of $-23 d B,-12 d B,-13 d B$ and $-14 d B$, respectively. Figure 19 depicts the measured and simulated $\left|S_{11}\right|$ of the proposed reconfigurable antenna within the four band mode.

Another three states which have the same notch bands, state 4 when PIN_2 and PIN_3 are ON and PIN_1 OFF, state 6 when PIN_1 and PIN_3 are ON and PIN_2 OFF, and state 8 when all PIN diodes are $\mathrm{ON}$, the proposed reconfigurable 


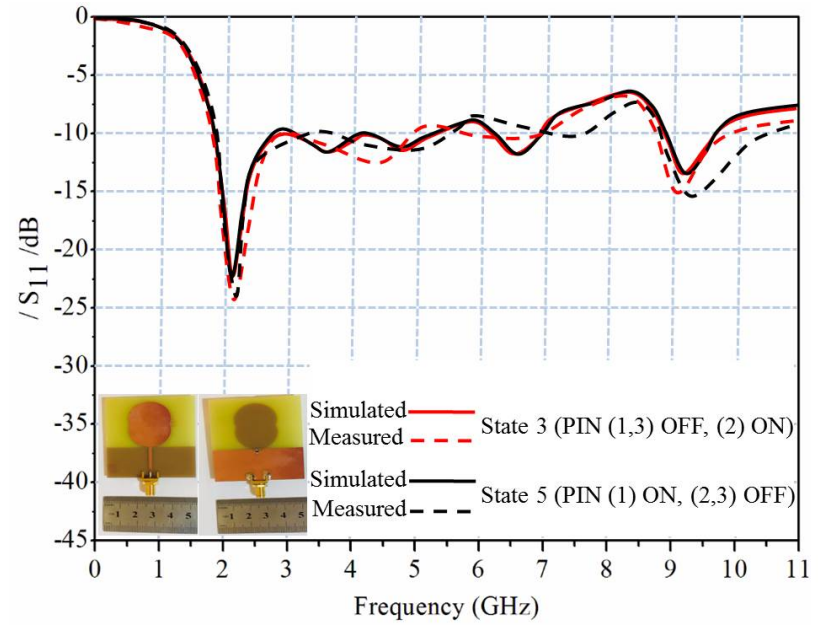

Fig. 19. The simulated and measured reflection coefficient for the operation states 3 and 5 .

antenna operates within three bands (1.86-2.65), (3-5.5), and (6.15-11.9) $G H z$ with a return loss of $-23 d B,-12 d B$, and -42 $d B$ respectively. Figure 20 depicts the measured and simulated $\left|S_{11}\right|$ of the proposed reconfigurable antenna within the three band mode.

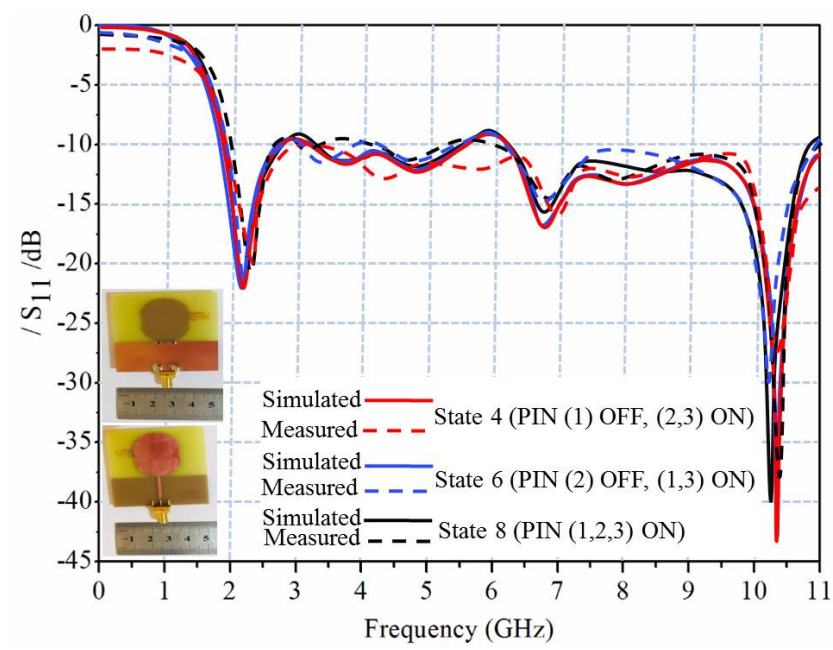

Fig. 20. The simulated and measured reflection coefficient for the operation states 4,6 and 8 .

From all the previous simulated and measured results, it is indicated that the proposed antenna has good ability to switch among UWB mode, dual, triple, and four band modes. The antenna radiation patterns are measured inside an RF chamber using a three-antenna method. The process is basically realized by conducting a standard dipole antenna at the frequency band of interest after calibrating the path losses inside the chamber. The simulated and measured radiation patterns in the E-plane and H-plane are depicted in Fig. 21 at $2.5 \mathrm{GHz}$. It is clear the radiation patterns of the proposed antenna display the same characteristics which are almost symmetrical in the Eplane and omnidirectional in the H-plane for the eight PIN diode states. Such stability in the radiation patterns for all the switches states in both E-plane and H-plane is a very desirable property in many applications [34]. This antenna patterns stability is due to selecting the PIN diodes position on the ground plane instead of the radiating patch element. However, due to manufacturing tolerance and the additional parasitic parameters caused by introducing several capacitors and bias wires, the simulated and measured radiation patterns are worse when integrating the PIN diodes on the antenna batch instead of the ground plane [34]. These eight radiation patterns under different biasing states exhibited certain similarities to each other, which confirmed the purpose of the proposed frequency reconfigurable antenna. Further, we noticed that for the eight switching states, the radiation pattern in the H-plane has an "8" shape, which indicates bidirectional radiation. This pattern is a typical radiation pattern like a conventional monopole antenna. The proposed reconfigurable antenna can have many possible applications in modern UWB and multi-functional mobile communication systems due to its excellent performance, ease of control and modification, and simple structure.

The current reconfigurable antenna is compared with the previously reported work [17]-[25]. Table III summarizes the comparison between this work and the literature. The proposed reconfigurable antenna has a very wide bandwidth as well as smaller size than [23]. The current design used only three PIN diodes compared with [23] and [25] for similar performances, which employs more than three PIN diodes. Another feature of the proposed antenna can switch among the UWB, dual, and triple band mode compared to the proposed antenna in [17]-[25], designed within a single mode. Thus, the present reconfigurable UWB antenna is more easily controlled among the desired band. The notch band characteristics can be realized by changing the PIN diode status rendering the antenna appropriate for UWB, dual, and triple operating bands. The low design complexity is another achievement in this work compared with suggested antenna design in [17]-[25]. The proposed antenna design does not have any slot on the radiation patch, resulting in UWB having notched band characteristics, therefore, the proposed technique does not significantly affect the proposed antenna's features. Finally, the proposed antenna performances unaffected due to the low ON resistance and insertion loss, as well as the PIN diode's easy biasing approach.

\section{CONCLUSion}

In this paper, we presented a compact UWB antenna that is low cost, light weight, and easy to control with an overall size of $50 \times 60 \times 1 \mathrm{~mm}^{3}$. The proposed antenna employs mounted three PIN diodes on the ground plane for achieving notched band characteristics. The reconfigurable antenna is fabricated on low-cost FR-4 substrate with $\epsilon_{r}=4.4$. It is designed to cover the entire UWB spectrum from $1.7 \mathrm{GHz}$ to $11 \mathrm{GHz}$. The reconfigurability of the frequency is accomplished by adjusting the effective electrical length of the proposed slot antenna. The reconfigurability is based on the insertion of three PIN diodes placed within a slotted ground plane. Due to the simplicity of the biasing circuit, it is simple to select 


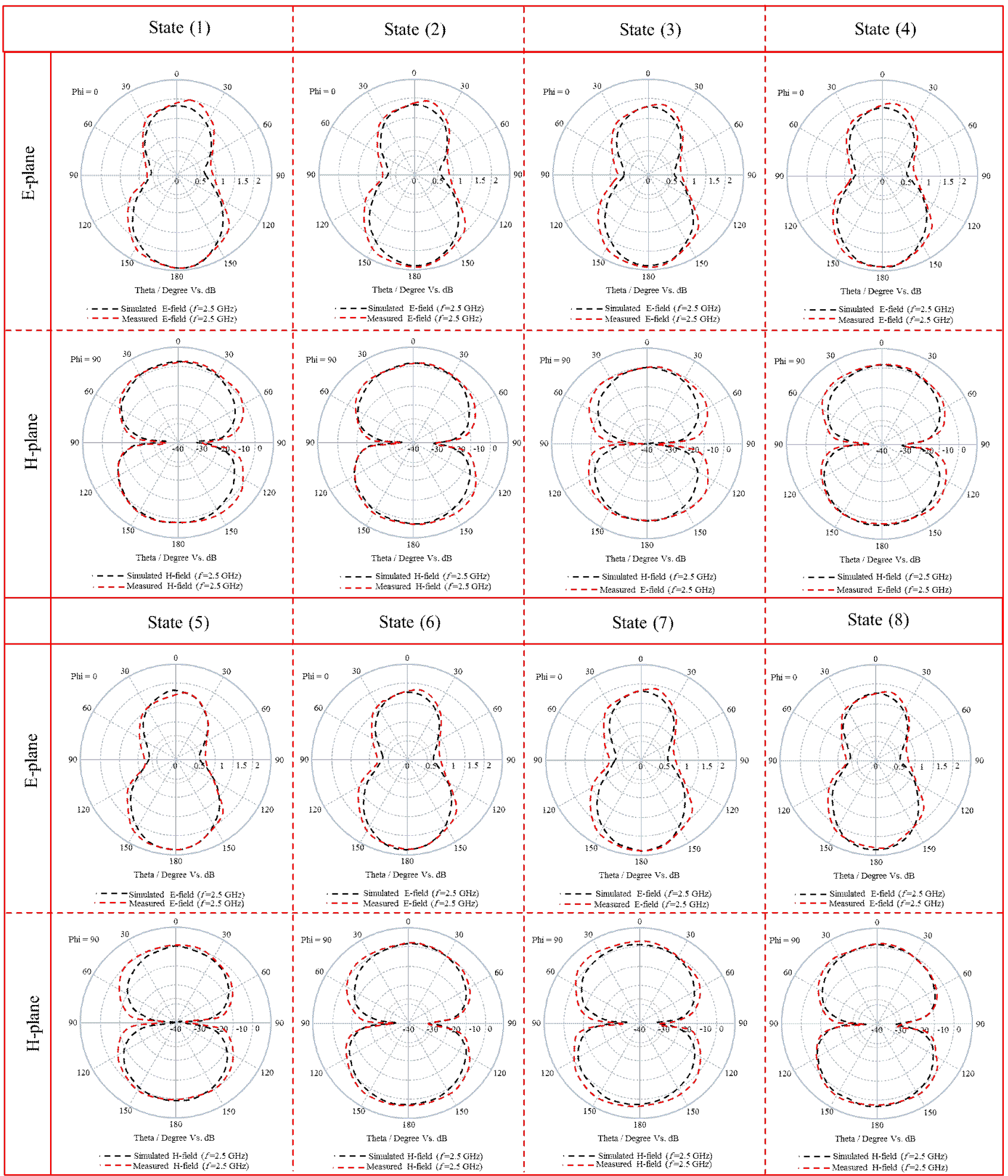

Fig. 21. Simulated and measured far-field radiation patterns at $2.5 \mathrm{GHz}$ in the E-plane and H-plane of the proposed UWB band notched antenna with all PIN diodes states. 
TABLE III

COMPARISON BETWEEN THE PROPOSED WORKS WITH RESPECT TO OTHER PUBLISHED RESULTS.

\begin{tabular}{|c|c|c|c|c|c|c|c|c|}
\hline Reference & Size $\left(\mathrm{mm}^{2}\right)$ & Reconfiguration & Substrate & RF switch & Notch bands & Rejected bands $(\mathrm{GHz})$ & No. of PIN diodes & Design Complexity \\
\hline$[17]$ & $30 \times 30$ & $\mathrm{~N}$ & FR4 & Varactor & $\mathrm{N}$ & $(2.7-7.1)$ & 1 & Moderate \\
\hline$[18]$ & $48 \times 7.47$ & Frequency & TLY-3-0450C5 & MEMS & $\mathrm{N}$ & $(4.8-7.4)$ & $\mathrm{N}$ & Moderate \\
\hline$[19]$ & $10 \times 12$ & Frequency & RO4350B & Varactor & Dual & $1.2-1.65$ & 1 & High \\
\hline$[20]$ & $50 \times 50$ & Frequency & FR4 & FET & $\mathrm{N}$ & $2.4,3.3,4.2,5.4$ & $\mathrm{~N}$ & High \\
\hline [21] & $33 \times 16$ & Frequency & FR4 & $\mathrm{N}$ & single/dual & $2.1,2.4,3.5,4.15,4.8,5.2$ & $\mathrm{~N}$ & Moderate \\
\hline [22] & $50 \times 50$ & Pattern & RO3006 & PIN & $\mathrm{N}$ & $5.2,5.8,6.4$ & 2 & High \\
\hline [23] & $100 \times 100$ & Polarization & RT/duroid 5880 & PIN & Dual & $2.53,2.44$ & 5 & High \\
\hline$[25]$ & $25 \times 25$ & Frequency & FR4 & PIN & Miltiband & $3.85,4.14,4.43,4.91,6.01$ & 5 & High \\
\hline This work & $50 \times 60$ & Frequency & FR4 & PIN & Single, dual, triple, UWB & $(2.77-3.3),(5.51-6.25)$ and $(10.87-20)$ & 3 & Low \\
\hline
\end{tabular}

the position to connect the biasing circuit. There is little impact on antenna bandwidth, gain, and radiation efficiency. The proposed PIN diode as a switching device is mounted within the ground plane instead of the antenna patch body. The main reason to choose three PIN diodes as switches is to change the proposed antenna's effective electrical length to achieve frequency reconfigurability. By switching the state of the PIN diode changes between forward and backward, you may get a double, triple, or UWB band mode. When all the switches are OFF, the proposed antenna is in a UWB operation band (1.85-10.9) $G H z$. The proposed antenna operates in dual band mode 2.7-3.5 $\mathrm{GHz}$ and 5.4-6.46 $\mathrm{GHz}$, when all switches are ON. The other six diode conditions allow for the operating band 2.7-3.5, 5.4-6.4, 7.5-8.4, and 8.87-10.05 $G H z$. The conventional monopole antenna is operating over the UWB. In this paper, the proposed antenna based on the PIN diodes is simulated, fabricated, measured, and tested. The simulated and measured results agree with the terms of $\left|S_{11}\right|$ spectra and radiation patterns for the proposed UWB antenna. The inclusion of the PIN diodes for the frequency notching on the antenna ground plane without impacting the antenna performance, which allowed more flexibility in the design of our antenna. The proposed reconfigurable monopole antenna with UWB, dual and triple operating bands is a good candidate for wireless applications such as WiFi and WiMAX. The proposed antenna could serve as an effective multimode application such as UWB and military reconfigurable frequency antennas.

\section{ACKNOWLEDGMENT}

The research reported in this paper was supported by the BME NC TKP2020 grant of NKFIH Hungary. The author Yahiea Al Naiemy would like to record his indebtedness to Tempus Public Foundation Stipendium Hungaricum program and University of Diyala - Iraq for their support for his $\mathrm{PhD}$ scholarship.

\section{REFERENCES}

[1] Row, Jeen-Sheen;You-Heng, Wei. Wideband reconfigurable crosseddipole antenna with quad-polarization diversity. IEEE Transactions on Antennas and Propagation 2018, 66.4, 2090-2094. DOI: 10.1109/TAP.2018.2800785

[2] Ojaroudi, Parchin; Naser, et al. Recent developments of reconfigurable antennas for current and future wireless communication systems. Electronics 2019, 8.2, 128. DoI: 10.3390/electronics 8020128

[3] Tang, Ming-Chun; et al. Compact, frequency-reconfigurable filtenna with sharply defined wideband and continuously tunable narrowband states. IEEE Transactions on Antennas and Propagation 2017, 65.10, 5026- 5034. DoI: 10.1109/TAP.2017.2736535
[4] Alnaiemy, Yahiea; Lajos, Nagy. Design of a Controllable Antenna Based on Embedded Differential PSK Modulation. Progress In Electromagnetics Research 2021, 90, 43-62. DOI: 10.2528/PIERB20100106

[5] Majid, Huda A.; et al. Frequency and pattern reconfigurable slot antenna. IEEE transactions on antennas and propagation 2014, 62.10, 5339-5343. DoI: 10.1109/TAP.2014.2342237

[6] Zhu, Ziqiang; et al. A flexible frequency and pattern reconfigurable antenna for wireless systems. Progress In Electromagnetics Research 2018, 76.10 , 63-70. Dor: 10.2528/PIERL18040401

[7] Han, Liping; et al. Design of frequency-and pattern-reconfigurable wideband slot antenna. International Journal of Antennas and Propagation 2018, 1-7, 2018. DoI: 10.1155/2018/3678018

[8] Iqbal, Amjad, et al. Frequency and pattern reconfigurable antenna for emerging wireless communication systems. Electronics 2019, 8.4, 407. DoI: 10.3390/electronics8040407

[9] Zhang, Zhuohang; Zhongming, Pan. Time domain performance of reconfigurable filter antenna for IR-UWB, WLAN, and WiMAX applications. Electronics 2019, 8.9, 1007. DOI: $10.3390 /$ electronics 8091007

[10] Wu, Terence; et al. Switchable quad-band antennas for cognitive radio base station applications. IEEE Transactions on Antennas and Propagation 2010, 58.5, 1468-1476. DoI: 10.1109/TAP.2010.2044472

[11] Hamid, M, R.; et al. Switched-band Vivaldi antenna. IEEE transactions on antennas and propagation. 2011, 59.5, 1472-1480. DoI: 10.1109/TAP.2011.2122293

[12] Yadav, Ajay; Minakshi, Tewari; Rajendra, Prasad, Yadav. Pixel shape ground inspired frequency reconfigurable antenna. Progress In Electromagnetics Research. 2019, 89, 75-85. DOI: $10.2528 /$ PIERC18082102

[13] Tasouji, Nasrin; et al. A novel printed UWB slot antenna with reconfigurable band-notch characteristics. IEEE Antennas and wireless propagation letters. 2013, 12, 922-925. DoI: 10.1109/LAWP.2013.2273452

[14] Han, Liping; Jing, Chen; Wenmei, Zhang. Compact UWB monopole antenna with reconfigurable band-notch characteristics. International Journal of Microwave and Wireless Technologies. 2020, 12.3, 252258. DoI: $10.1017 / \mathrm{S} 1759078719001296$

[15] Oraizi, Homayoon; Nooshin, Valizade Shahmirzadi. Frequency-and time-domain analysis of a novel UWB reconfigurable microstrip slot antenna with switchable notched bands. IET Microwaves, Antennas and Propagation. 2017, 11.8, 1127-1132. DOI: 10.1049 /iet-map.2016.0009

[16] Li, Yingsong, et al. A small multi-function circular slot antenna for reconfigurable UWB communication applications. IEEE Antennas and Propagation Society International Symposium (APSURSI). 2014, 1, 834- 835. DoI: 10.1109/APS.2014.6904745

[17] Aghdam; Sajjad, Abazari. A novel UWB monopole antenna with tunable notched behavior using varactor diode. IEEE Anten- nas and Wireless Propagation Letters. 2014, 13, 1243-1246. DoI: 10.1109/LAWP.2014.2332449

[18] Saghati; Alireza, Pourghorban; Kamran, Entesari. A reconfigurable SIW cavity-backed slot antenna with one octave tuning range. IEEE transactions on antennas and propagation. 2013, 61.8, 3937-3945. DoI: 10.1109/TAP.2013.2263215

[19] Behdad, Nader; Kamal, Sarabandi. A varactor-tuned dual-band slot antenna. IEEE Transactions on Antennas and Propagation. 2006, 54.2, 401-408. DoI: 10.1109/TAP.2005.863373 
[20] Aboufoul, Tamer; Akram, Alomainy; Clive, Parini. Reconfiguring UWB monopole antenna for cognitive radio applications using GaAs FET switches. IEEE Antennas and Wireless Propagation Letters. 2012, 11, 392-394. DoI: 10.1109/LAWP.2012.2193551

[21] Shah, I. A.; et al. Design and analysis of a hexa-band frequency reconfigurable antenna for wireless communication. $A E U$ International Journal of Electronics and Communications. 2019, 98, 80-88. DoI: $10.1016 /$ j.aeue.2018.10.012

[22] Nikolaou, Symeon; et al. Pattern and frequency reconfigurable annular slot antenna using PIN diodes. AEU-International Journal of Electronics and Communications. 2006, 54.2, 439-448. DoI: 10.1109/TAP.2005.863398

[23] Kim, Boyon; et al. A novel single-feed circular microstrip antenna with reconfigurable polarization capability. IEEE Transactions on Antennas and Propagation. 2008, 56.3, 630-638. DoI: 10.1109/TAP.2008.916894

[24] Elwi, Taha A. Remotely controlled reconfigurable antenna for modern 5G networks applications. Microwave and Optical Technology Letters. 2020. DoI: 10.1002/mop.32505

[25] Singh, Prem Pal; et al. Frequency reconfigurable multiband antenna for IoT applications in WLAN, Wi-Max, and C-band. Progress In Electromagnetics Research. 2020, 102, 149-162. DOI: 10.2528/PIERC20022503

[26] Reddy, Bobbili Naga Balarami; et al. Design and analysis of wideband monopole antennas for flexible/wearable wireless device applications. Progress In Electromagnetics Research M. 2017, 62, 167-174. DOI: $10.2528 /$ PIERM17092107

[27] Mohandoss, Susila; et al. Fractal based ultra wideband antenna development for wireless personal area communication applications. AEU International Journal of Electronics and Communications. 2018, 93, 95- 102. Dor: 10.1016/j.aeue.2018.06.009

[28] Kingsly, Saffrine; et al. Multiband reconfigurable filtering monopole antenna for cognitive radio applications. IEEE Antennas and Wireless Propagation Letters. 2018, 17.8, 1416-1420. DOI: 10.1109/LAWP.2018.2848702

[29] Mohandoss, Susila; et al. On the bending and time domain analysis of compact wideband flexible monopole antennas. AEU-International Journal of Electronics and Communications. 2019, 101, 168-181. DoI: 10.1016/j.aeue.2019.01.015

[30] Studio, CST Microwave. URL http://www.cst.com/Content/Products/ MWS.. Overview. aspx. 2017.

[31] Rahim, M. K. A.; et al. Frequency reconfigurable antenna for future wireless communication system. 2016 46th European Microwave Conference (EuMC). IEEE 2016, 2016. DoI: $10.1109 / \mathrm{EuMC} .2016 .7824506$

[32] Lakrit, Soufian; et al. Compact UWB flexible elliptical CPWfedantenna with triple notch bands for wireless communications. International Journal of $R F$ and Microwave Computer-Aided Engineering. 2020, 30.7, 2020. DoI: 10.1002/mmce.22201

[33] www.alldatasheet.com, datasheetpdf. URL http://www.alldatasheet. com/INFINEON./396627,BAR63-02V.

[34] Al Gburi, Mohaimen; and Muhammad Ilyas. A Novel Design Reconfigurable Antenna Based on the Metamaterial for Wearable Applications. Journal of Physics: Conference Series. 2021, Vol. 1973. No. 1. IOP, 2021. DoI: 10.1088/1742-6596/1973/1/012042

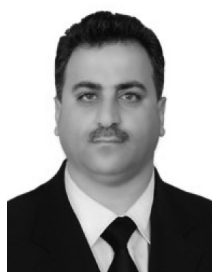

Yahiea Alnaiemy He received his Bachelor's Degree in Electrical Engineering from AL-Mustansiriyah University, Faculty Of Engineering in 1998. He continued his graduate studies by joining the Iraq Commission for Computers and Informatics, where he received a Higher Diploma in Information Systems in 2001. He enrolled at Diyala University as an instructor in communication Engineering, electrical power, computer, and physics departments. In 2009 , he granted a scholarship to complete his master's degree in electrical engineering at the University of Arkansas at Little Rock, USA. He got his MSc in Wireless Communications from UALR, USA, in 2012. While completing his graduate degree, his research effort has been in the area of antennas and microwave material characterization. In 2017, he granted a scholarship to complete his Ph.D. in electrical engineering at BME, Hungary. His current research areas include UWB antennas, EBG structures, metamaterial, GPS, implantable wireless systems, nanoscale microwave devices, reconfigurable antennas, and RF power harvesting. He has been an IEEE Member and reviewer in several international journals and conferences since 2011.

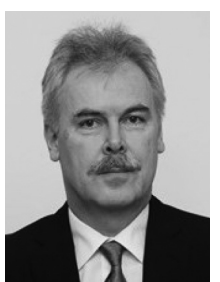

Lajos Nagy $\mathrm{He}$ received the Engineer option Communication) and $\mathrm{PhD}$ degrees, both from the Budapest University of Technology and economics (BME), Budapest, Hungary, in 1986 and 1995 , respectively. He joined the department of Microwave Telecommunications (now Broadband Infocommunications and Electromagnetic Theory) in 1986 , where he is currently an associate professor. He has been the head of department of Broadband Infocommunications and Electromagnetic Theory in 2007. He is a lecturer on graduate and postgraduate courses at BME on Antennas and radiowave propagation, Radio system design, Adaptive antenna systems and Computer Programming. His research interests include antenna analysis and computer aided design, electromagnetic theory, radiowave propagation, communication electronics, signal processing and digital antenna array beamforming, topics where he has produced more than 100 different book chapters and peer-reviewed journal and conference papers. Member of Hungarian Telecommunication Association, official Hungarian Member and Hungarian Committee Secretary of URSI, Chair of the IEEE Chapter AP/ ComSoc/ED/MTT 